\title{
ANÁLISE ESTRUTURAL E ESPACIAL DO COMPONENTE ARBÓREO COMO ELEMENTO DE PAISAGEM TURISTÍCA
}

\section{STRUCTURAL AND SPATIAL ANALYSIS OF THE ARBORING COMPONENT AS A TOURIST LANDSCAPE ELEMENT}

CHARLES COSTA COELHO, M.SC. FURB

MARCELO DINIZ VITORINO, Dr. FURB

BRUNO JUAN GUEDES RODE | FURB

ANA CAROLINA BOSCHETTI, M.SC. |FURB

PAULO AUGUSTO GARBUGIO DA SILVA, M.SC.| UFSC

TATIELE ANETE BERGAMO FENILLI, Dra. | FURB

MARIA RAQUEL KANIESKI, Dra. | UDESC

KRISTIANA FIORENTIN DOS SANTOS, Dra. | FURB

\begin{abstract}
RESUMO
O objetivo do trabalho foi realizar uma caracterização do patrimônio arbóreo-arbustivo das vias e praças públicas das cidades de São Joaquim, Urubici e Urupema, Santa Catarina, Brasil. Para cada indivíduo mensurado foi realizada uma avaliação quali-quantitativa, conforme a metodologia do Grau de Atenção para Árvores Urbanas - GAAU. Posteriormente, foi calculado o índice de diversidade de arborização, para as principias avenidas dos municípios de estudo. Em São Joaquim, Urupema e Urubici foram observados 462, 236 e 201 indivíduos, distribuídos em 35, 26 e 23 espécies, respectivamente. Sobre a origem das espécies, a maior parte delas são exóticas, ou seja, $86,6 \%, 61,5 \%$ e $66,7 \%$ das espécies encontradas nos municípios citados acima. Houve uma frequência maior no grau de atenção "Baixo", sendo 93,0\% para Urupema, 87,7\% Urubici e 74,2\% São Joaquim, seguido pelo grau de atenção "Médio" com 6,9\%, 11,8\% e 25,1\% seguindo a mesma ordem das cidades apresentada anteriormente. Já os menores valores encontrados pertencem ao grau de atenção "Alto", encontrado apenas em duas das três cidades de estudo, sendo São Joaquim com 0,6\% e Urubici com $0,4 \%$. Quando aplicado os índices de diversidade observa-se que das oito ruas avaliadas, sete estão com valores abaixo do que é considerada uma diversidade satisfatória (2,45), sendo necessários novos plantios e enriquecimento com novas espécies nativas da região. Logo, com a adição do número recomendado de espécies, o índice de todas as ruas teria uma melhoria, proporcionando desta forma maior qualidade ambiental para a cidade, bem como, a melhoria da qualidade de vida de seus habitantes.
\end{abstract}

PALAVRAS CHAVE: Arborização viária; Diversidade; Espécies nativas; Planejamento urbano.

\begin{abstract}
The purpose of this work was to investigate of the tree-shrub composition of road afforestation and public squares in the cities of São Joaquim, Urubiciand Urupema, in the state of Santa Catarina. A visual assessment was carried out applying the Degree of Attention to Urban Trees (GAAU), amethodology developed to carry out the presentwork. Afforestation diversity indexes were determined. In total, 899 tree-shrub components were cataloged, distributed in 57 different species, presents in the three studied areas. There was a higher frequency in the "Low" degree of attention, 93.0\% for Urupema, 87.7\% Urubici and 74.2\% São Joaquim, followed by the "Medium" degree of attention with $6.9 \%, 11.8 \%$ and $25.1 \%$ following the same order of cities presented above. The lowest values found belong to the "High" degree of attention, found only in two of the threestudied cities, being São Joaquim with $0.6 \%$ and Urubici with $0.4 \%$. No individuals were found to justify the "Priority" level of attention. The diversity indexes showed that from eight streets evaluated, seven has unsatisfactory diversity (2.45), being new planting and enrichment with new native species from the region necessary.
\end{abstract}

KEY WORDS: Road afforestation; Diversity; Native species; Urban planning. 


\section{INTRODUÇÃO}

As florestas urbanas são um elemento fundamental para garantir a qualidade de vida nas cidades (DEMUZERE et al., 2014; NORTON et al., 2015). As árvores presentes nas áreas urbanas desempenham uma série de benefícios fundamentais (NOWAK et al., 1996), tais como a ciclagem da água e de nutrientes, diminuição da temperatura, sequestro de carbono, além de diversos serviços ecossistêmicos (GAGE e COOPER, 2017).

No entanto, esses serviços podem ser comprometidos pelo ataque de pragas e patógenos, atribuídos a falta de manejo ou a sua realização de forma incorreta, trazendo como consequência vários danos as plantas e colocando em risco a comunidade arbórea (REDIN et al., 2010; SJÖMAN et al., 2012; SANTOS et al., 2015). Muitos desses danos são ocasionados durante a poda, embora ela seja extremamente necessária, principalmente nas árvores localizadas nas calçadas e canteiros centrais, de modo a evitar uma gama de problemas, além de garantir a visibilidade dos pedestres e motoristas, e a liberação de linhas de energia (FINI et al., 2015; GROHMANN et al., 2018).

Dentre os principais problemas que devem ser identificados e manejados pelos gestores públicos, destaca-se a avaliação fitossanitária das árvores, que deve ser feita cautelosamente para identificar risco de queda, proliferação de pragas e doenças à flora do meio urbano e de seu entorno (MOURA DE SOUSA et al., 2013).

Porém, ainda são poucas as ações realizadas para a melhoria das condições ambientais da infraestrutura verde urbana, dentre elas, a conscientização ambiental e o inventário florestal, que em inúmeros casos, os gestores sentem dificuldade de gerir, devido as suas próprias propostas, por vezes, não atendendo as necessidades coletivas da população (SOUZA, 2011; VIBRANS et al., 2012).

Estudos a respeito do tema são escassos na região da Serra Catarinense, com destaque para os trabalhos de Pinheiro et al. (2009) na cidade de São José do Cerrito, Santos et al. (2013) com o inventário da arborização viária da cidade de Lages, e Oliveira et al. (2019) com o levantamento da Praça Centenário de Curitibanos.

Diante do exposto, é necessário que os gestores se capacitem progressivamente para suprir as necessidades pontuais da arborização de cada cidade, visto que há uma lacuna na gestão desse recurso tão elementar (ARAÚJO et al., 2013). Desse modo o propósito do trabalho foram: (i) realizar uma avaliação quali-quantitativo das espécies arbóreas-arbustiva da arborização viária e das praças públicas, presentes nas cidades de São Joaquim, Urubici e Urupema (SC); (ii) avaliar os índices de diversidade e distribuição espacial de arborização em cada cidade e (iii) sugerir meIhorias para arborização urbana com indicação de espécies nativas da região com o foco de fomentar o turismo.

\section{MATERIAL E MÉTODOS}

\section{1 Área de Estudo}

O trabalho foi realizado na Região do Planalto Catarinense, nas cidades de São Joaquim, Urubici e Urupema (Figura 1). O clima da região é caracterizado como $\mathrm{Cfb}$, com temperatura média anual de $13,5-14,0^{\circ} \mathrm{C}$ e a precipitação média anual varia entre 1,707 a 1,740 mm (ALVARES et al., 2013).

Os solos são de origem basáltica, sendo o Cambissolo o tipo predominante (SANTOS et al., 2018). A vegetação é formada pelas florestas de Araucárias, classificada como Floresta Ombrófila Mista (IBGE, 2018a, b, c).
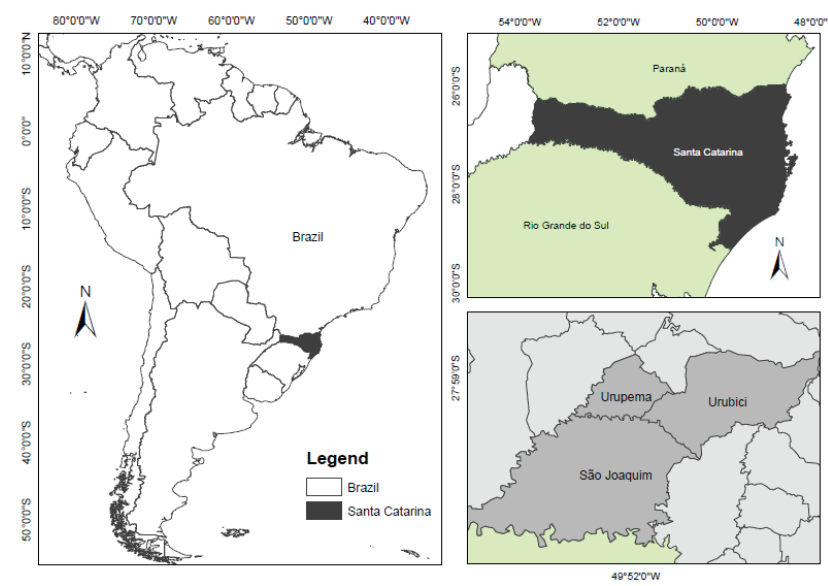

Figura 01 - Localização geográfica da área de estudo. Fonte: Autores

\subsection{Coleta e Processamento de Dados}

No município de São Joaquim foi realizada a amostragem estratificada dos indivíduos arbóreo-arbustivo, envolvendo as principais vias de acesso da cidade, e as praças públicas do centro com maior intensidade de circulação de pedestres e veículos. Já nos municípios de Urupema e Urubici foi realizado um censo, ou seja, todos os indivíduos encontrados na arborização viária e praças públicas foram mensurados.

Para cada indivíduo mensurado foi realizada uma avaliação quali-quantitativa, conforme a metodologia do Grau de Atenção para Árvores Urbanas - GAAU. Essa metodologia é baseada em uma análise visual e foi elaborada como um instrumento de avaliação das condições das árvores urbanas em $360^{\circ} \mathrm{com}$ seu entorno. A avaliação foi dividida em quatro categorias: avaliação da copa ( $A C$ ), avaliação do tronco (AT), avaliação da base do tronco (AB) e avaliação do alvo (AA). 


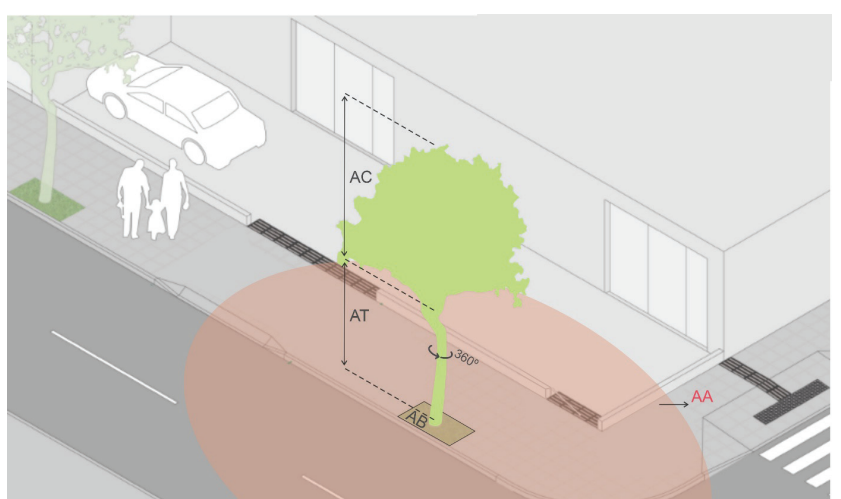

Figura 02 - Esquema representando as dimensões da avaliação empregada no inventário florestal urbano, $0 \mathrm{GAAU}$, aplicado na arborização viária e arborização de praças públicas nas cidades de São Joaquim, Urubici e Urupema (SC).

Fonte: Adaptado do IPUF, 2019.

Para coleta de dados a campo elaborou-se uma base de dados em formato SIG (Sistema de Informações Geográficas). Esta base de dados foi realizada no software $\operatorname{ArcGIS}^{\circledast}$ online. Após a criação da base, foi instalado em aparelho celular o aplicativo Collector for $\operatorname{ArcGIS}^{\circledR}$, o qual permite realizar o download do mapa onde será realizado o inventário e inserir as informações a campo direto no apareIho, além de possibilitar a coleta de dados de modo offline.

Posteriormente, no Microsoft Office Excel ${ }^{\circledR}$, foi calculado o índice de diversidade de Odum (d1), para as principias avenidas dos municípios de estudo (ODUM, 2001).

$$
\begin{array}{r}
d 1=(F S-1) \\
\ln N
\end{array}
$$

Em que:

d 1 = Índice de diversidade;

$S=$ Número total de espécies;

$N=$ Somatório do número de indivíduo encontrado no inventário.

a) Espécies a serem adicionadas ( $\mathrm{S}$ ad)

Este índice demonstra a necessidade de implantação ou remoção de espécies nas vias públicas, considerando que não se exceda a 10,0\% de uma espécie numa paisagem urbana. Este índice é calculado conforme Filho e Bortoleto, 2005.

$$
S_{a d}=\left[d_{\text {min }}-d_{1} * \ln \left(N_{\text {exist. }}-N_{\text {pot. }}\right)\right]+f c
$$

Em que:

$\mathrm{S}$ ad = Quantidade de espécies a serem adicionadas;

$d$ mín = Índice de Odum teórico mínimo $(2,45)$;

$d 1$ = Índice de Odum atual (calculado no inventário);

Nexist. $=$ Somatória do número de espécies existentes (obtidas no inventário);

Npot. = Somatória do número de indivíduos potenciais; $f c=$ Fator de correção.
Com o objetivo de evitar que o índice resultante do novo plantio seja inferior que dmín, é adotado o fator de correção de 5,5 (fc). Para calcular o número de árvores potenciais em uma via pública, será adotado o cálculo de Filho e Bortoleto (2005), o qual é considerado que o número de indivíduos ideal por km é de 133 indivíduos. Levando em conta, os dois lados da via, e um espaçamento de 15,0 m entre cada árvore (BIZ, 2015).

Em que:

$$
N_{\text {pot }}=133-N_{\text {exist. }}
$$

$\mathrm{N}$ pot. = Somatória do número de indivíduos potenciais; 133 = Número de árvores potenciais em 1 km, com espaçamento de $15 \mathrm{~m}$ entre indivíduos.

b) Índice de Diversidade Futura de Odum (dfut)

O $d$ fut é um indicador de diversidade futura, após a adição de novas espécies (FILHO; BORTOLETO, 2005), sendo calculado pela seguinte equação:

$$
d_{\text {fut }}=\left[\left(\left(d_{\text {atual }}+S\right)-1\right) / \ln \left(N_{\text {pot. }}+N_{\text {exist. }}\right)\right]
$$

Em que:

$d$ fut = Índice de diversidade futura de Odum;

d atual = Índice de diversidade de Odum atual;

$S=$ Número de espécies;

$N$ pot. $=$ Número de árvores potenciais;

Nexist. $=$ Número de árvores existentes

Para análise da distribuição e densidade das árvores mapeadas no estudo, foi aplicado o estimador de densidade de Kernel, no software ArcMap versão 10.4.1. Esta análise é uma técnica não paramétrica para estimação de curvas de densidades no qual cada observação é ponderada pela distância em relação ao núcleo (BRASIL, 2007). Após a geração das imagens de densidade, foram aplicadas cinco classes de densidade, conforme proposto por Mayer et al. (2014) sendo: muito alto, alto, médio, baixo e muito baixo.

\section{RESULTADOS E DISCUSSÕES}

Nos municípios estudados foram constatados 899 indivíduos arbóreo-arbustivos, distribuídos em 57 espécies. Especificamente em São Joaquim, Urupema e Urubici foram observados 462, 236 e 201 indivíduos, distribuídos em 35, 26 e 23 espécies, respectivamente (Quadro 1).

No geral foram encontradas 26 famílias botânicas, destacando-se Rosaceae $(n=6)$, Cupressaceae e Sapindaceae com maior quantidade de espécies, ou seja, 6,5 e 5, respectivamente. Conforme observado em outros estudos realizados no Brasil, o número de famílias constatadas no presente trabalho é inferior comparado a outras cidades brasileiras, como Maringá-PR com 37 
famílias (SAMPAIO, 2006), Santa Maria-RS com 30 famílias (ANDREATT et al., 2011) e Imperatriz-MA com 27 famílias botânicas (SILVA et al., 2018).

Em contrapartida, em relação a cidades pertencentes a região do planalto catarinense, o número de famílias encontradas por área de estudo, ou seja, 18, 16 e 14 em São Joaquim, Urubici, e Urupema, respectivamente, observa-se um valor próximo ou superior ao encontrado, sendo relatado por Santos et al. (2013) 13 famílias na cidade de Lages, 8 em São José do Cerrito (PINHEIRO et al., 2009) e 29 em Curitibanos (OLIVEIRA et al., 2019).
Sobre a origem das espécies, a maior parte delas são exóticas, ou seja, 86,6\%,61,5\% e 66,7\% das espécies encontradas em São Joaquim, Urubici e Urupema, respectivamente. Dentre elas, seis são consideradas invasoras, conforme a Lei no 14.675/2009 - Lei Oficial de Espécies Exóticas Invasoras no Estado de Santa Catarina e com a base de dados Nacional do Instituto Hórus de Desenvolvimento e Conservação Ambiental (2016), sendo elas: Cotoneaster franchetii B.; Eriobotrya japonica (Thunb.) Lindl.; Ligustrum lucidum W. T. Aiton; Melia azedarach L.; Pinus elliottii Engelm. e Pinus taeda L.

\begin{tabular}{|c|c|c|c|c|c|c|c|c|}
\hline \multirow{3}{*}{ Família } & \multirow{3}{*}{ Nome científico } & \multirow{3}{*}{ Origem } & \multicolumn{6}{|c|}{ Cidade } \\
\hline & & & \multicolumn{2}{|c|}{ São Joaquim } & \multicolumn{2}{|c|}{ Urubici } & \multicolumn{2}{|c|}{ Urupema } \\
\hline & & & $\mathbf{n}$ & FR & n & FR & $\mathbf{n}$ & FR \\
\hline Altingiaceae & Liquidambar orientalis Mill. & E & 57 & 11,5 & - & - & - & - \\
\hline Anacardiaceae & Schinus terebinthifolia Raddi & $\mathrm{N}$ & - & - & 4 & 1,6 & 10 & 4,9 \\
\hline Araucariaceae & Araucaria angustifolia (Bertol.) Kuntze & $\mathrm{N}$ & 9 & 1,9 & 4 & 1,6 & 29 & 14,4 \\
\hline \multirow[t]{3}{*}{ Arecaceae } & Butia catarinensis Noblick \& Lorenzi & $\mathrm{N}$ & 3 & 0,6 & 2 & 0,8 & 2 & 1,0 \\
\hline & Syagrus romanzoffiana (Cham.) Glassman & $\mathrm{N}$ & 1 & 0,2 & 4 & 1,6 & - & - \\
\hline & Trachycarpus fortunei (Hook.) H. Wendl. & $\mathrm{E}$ & 6 & 1,3 & 11 & 4,6 & - & - \\
\hline Asparagaceae & Yucca gigantea Lem. & $\mathrm{E}$ & - & - & 19 & 8,0 & - & - \\
\hline Asteraceae & Moquiniastrum polymorphum (Less.) G. Sancho & $\mathrm{N}$ & 1 & 0,2 & - & - & - & - \\
\hline \multirow[t]{3}{*}{ Bignoniaceae } & Handroanthus albus (Cham.) Mattos & $\mathrm{N}$ & 9 & 1,9 & 4 & 1,6 & - & - \\
\hline & Handroanthus chrysotrichus (Mart. ex A.DC.) Mattos & $\mathrm{N}$ & 2 & 0,4 & 4 & 1,6 & - & - \\
\hline & Handroanthus heptaphyllus (Vell.) Mattos & $\mathrm{N}$ & - & - & 1 & 0,4 & 1 & 0,5 \\
\hline \multirow[t]{5}{*}{ Cupressaceae } & Chamaecyparis lawsoniana (A. Murr.) Parl & $\mathrm{E}$ & 4 & 0,8 & - & - & 7 & 3,4 \\
\hline & Chamaecyparis pisifera var. filifera Hartwig \& Rümpler & $\mathrm{E}$ & 11 & 2,2 & 5 & 2,1 & 10 & 4,9 \\
\hline & Cupressus sempervirens $L$. & $\mathrm{E}$ & - & - & 50 & 21,1 & 48 & 23,8 \\
\hline & Juniperus chinensis "variegata" $L$. & $\mathrm{E}$ & 1 & 0,2 & - & - & - & - \\
\hline & Thuja occidentalis $L$. & $\mathrm{E}$ & 30 & 6,0 & 9 & 3,8 & 15 & 7,4 \\
\hline Dicksoniaceae & Dicksonia sellowiana Hook & $\mathrm{N}$ & 3 & 0,6 & - & - & 17 & 8,4 \\
\hline \multirow[t]{3}{*}{ Fabaceae } & Erythrina falcata Benth. & $\mathrm{N}$ & 1 & 0,2 & - & - & - & - \\
\hline & Mimosa scabrella Benth. & $\mathrm{N}$ & 1 & 0,2 & - & - & - & - \\
\hline & Peltophorum dubium (Spreng.) Taub. & $\mathrm{N}$ & - & - & - & - & 1 & 0,5 \\
\hline Ginkgoaceae & Gingko Biloba L. & $\mathrm{E}$ & 2 & 0,4 & - & - & - & - \\
\hline Lythraceae & Lagerstroemia indica L. & $\mathrm{E}$ & 1 & 0,2 & 16 & 6,7 & 1 & 0,5 \\
\hline \multirow[t]{3}{*}{ Magnoliaceae } & Cedrela fissilis Vel. & $\mathrm{N}$ & 2 & 0,4 & - & - & - & - \\
\hline & Liriodendron tulipifera L. & $\mathrm{E}$ & 30 & 6,0 & - & - & - & - \\
\hline & Magnolia x soulangeana var. alba-superba & $\mathrm{E}$ & 2 & 0,4 & - & - & - & - \\
\hline Meliaceae & Melia azedarach L. & E & - & - & 2 & 0,8 & - & - \\
\hline Moraceae & Ficus cestrifolia Schott ex Spreng. & $\mathrm{N}$ & - & - & 1 & 0,4 & - & - \\
\hline \multirow[t]{4}{*}{ Myrtaceae } & Acca sellowiana (O. Berg) Burret & $\mathrm{N}$ & 6 & 1,2 & 8 & 3,3 & 9 & 4,4 \\
\hline & Callistemon speciosus (Sims) DC. & $\mathrm{E}$ & - & - & 1 & 0,4 & - & - \\
\hline & Eugenia uniflora $L$. & $\mathrm{N}$ & - & - & 1 & 0,4 & - & - \\
\hline & Psidium cattleianum Sabine & $\mathrm{N}$ & - & - & 2 & 0,8 & - & - \\
\hline \multirow[t]{2}{*}{ Oleaceae } & Ligustrum lucidum W. T. Aiton & $\mathrm{E}$ & 20 & 4,0 & 68 & 28,8 & 22 & 10,9 \\
\hline & Ligustrum sinense Lour. & E & 1 & 0,2 & - & - & - & - \\
\hline
\end{tabular}




\begin{tabular}{|c|c|c|c|c|c|c|c|c|}
\hline & Olea europaea L. & E & - & - & 1 & 0,4 & - & - \\
\hline Paulowniaceae & Paulownia tomentosa (Thunb.) Steud. & E & - & - & 1 & 0,4 & - & - \\
\hline \multirow[t]{2}{*}{ Pinaceae } & Pinus elliotti Engelm & $\mathrm{E}$ & 2 & 0,2 & - & - & - & - \\
\hline & Pinus taeda $L$. & $\mathrm{E}$ & 3 & 0,6 & - & - & - & - \\
\hline Platanaceae & Platanus $x$ acerifolia (Aiton.) Willd & $\mathrm{E}$ & 45 & 9.0 & - & - & - & - \\
\hline \multirow[t]{2}{*}{ Podocarpaceae } & Podocarpus lambertii Klotzsch ex Endl. & $\mathrm{N}$ & 1 & 0,2 & - & - & - & - \\
\hline & Podocarpus macrophyllus (Thunb.) Doce & $\mathrm{E}$ & - & - & 4 & 1,6 & 6 & 2,9 \\
\hline \multirow[t]{9}{*}{ Rosaceae } & Chaenomeles speciosa (Doce) Nakai & $\mathrm{E}$ & - & - & - & - & 1 & 0,5 \\
\hline & Cotoneaster franchetii Bois & E & 1 & 0,2 & - & - & 2 & 1,0 \\
\hline & Eriobotrya japônica (Thunb.) Lindl. & $\mathrm{E}$ & - & - & 3 & 1,2 & - & - \\
\hline & Malus sp. & $\mathrm{E}$ & 2 & 0,4 & - & - & - & - \\
\hline & Prunus avium $L$. & $\mathrm{E}$ & - & - & - & - & 10 & 4,9 \\
\hline & Prunus persica L. Batsch & $\mathrm{E}$ & 5 & 1,0 & - & - & 1 & 0,5 \\
\hline & Prunus serrulata Lindl. & $\mathrm{E}$ & 107 & 21,4 & 2 & 0,8 & - & - \\
\hline & Pyrus communis $L$. & $\mathrm{E}$ & - & - & - & - & 1 & 0,5 \\
\hline & Spiraea cantoniensis Lour. & $E$ & - & - & - & - & 4 & 1,9 \\
\hline \multirow[t]{3}{*}{ Salicaceae } & Populus alba L. & $\mathrm{E}$ & - & - & 2 & 0,8 & - & - \\
\hline & Populus nigra L. & $\mathrm{E}$ & - & - & - & - & 1 & 0,5 \\
\hline & Salix babylonico $L$. & $\mathrm{E}$ & - & - & - & - & 1 & 0,5 \\
\hline \multirow[t]{4}{*}{ Sapindaceae } & Acer forrestii Diels & $\mathrm{E}$ & 24 & 4,8 & - & - & - & - \\
\hline & Acer palmatum Atropurpureum & $\mathrm{E}$ & 14 & 2,8 & - & - & - & - \\
\hline & Acer palmatum Thunb. & $\mathrm{E}$ & 28 & 5,6 & - & - & - & - \\
\hline & Sapindus saponaria L. & $\mathrm{N}$ & - & - & - & - & 2 & 1,0 \\
\hline Theaceae & Camellia japônica L. & $\mathrm{E}$ & 4 & 0,8 & 10 & 4,2 & - & - \\
\hline- & Indivíduos mortos & - & 23 & 4,6 & 1 & 0,4 & - & - \\
\hline Total & - & - & 462 & 100 & 236 & 100 & 201 & 100 \\
\hline
\end{tabular}

Quadro 01 - Espécies arbóreo-arbustivas classificadas por família, gênero e espécie botânica. Período de julho a novembro de 2019. Fonte: Autores

A classe de altura com maior abundância foi de pequeno porte $(<6 \mathrm{~m})$, seguida pelas classes de médio e grande porte. Sobre a classe de pequeno porte, foram constatados 367 indivíduos em São Joaquim, 172 em Urubici e 169 em Urupema. Na classe de médio porte (6 a $12 \mathrm{~m}$ ) foram observados 75, 53 e 31 indivíduos distribuídos em São Joaquim, Urubici e Urupema, respectivamente. Em contrapartida, o menor número foi encontrado na classe de grande porte $(<12 \mathrm{~m})$, sendo que em Urupema foi encontrado apenas um único indivíduo. Esses valores podem ser resultantes das condições edafoclimáticas da região, onde as espécies demoram mais para se desenvolver pelas condições de baixas temperaturas, principalmente nas estações outono-inverno.

Em relação à escolha de espécies, nota-se que a população e gestores municipais das três cidades dão preferência a espécies de pequeno porte e caducifólias, para que a luz solar possa incidir direto sobre a casa aquecendo nos períodos mais frios do ano.
Os valores encontrados para o GAAU são apresentados na Quadro 2. Nota-se que nas cidades estudadas houve uma frequência maior no grau de atenção "baixo", sendo 93,0\% para Urupema, 87,7\% Urubici e 74,2\% São Joaquim, seguido pelo grau de atenção "médio" com 6,9\%, 11,8\% e $25,1 \%$ seguindo a mesma ordem das cidades acima. Já os menores valores encontrados pertencem ao grau de atenção "alto", encontrado apenas em duas das três cidades de estudo, sendo São Joaquim com $0,6 \%$ e Urubici com $0,4 \%$.

Não foram encontrados indivíduos que justificasse o grau de atenção "prioritário". É importante ressaltar que esta classe determina que uma ação deva ser realizada de forma imediata no indivíduo, amenizando qualquer conflito ou ataque de praga/doenças que a árvore esteja sofrendo, bem como eliminando riscos de queda. 


\begin{tabular}{|l|l|l|l|l|l|l|}
\hline \multirow{2}{*}{$\begin{array}{l}\text { GRAU DE } \\
\text { ATENÇÃO PARA } \\
\text { ÁRVORES URBANAS } \\
\text { - GAAU }\end{array}$} & \multicolumn{2}{l}{ CIDADE } \\
\cline { 2 - 9 } & \multicolumn{2}{l}{\begin{tabular}{l}
\multicolumn{2}{l}{ São } \\
Joaquim
\end{tabular}} & \multicolumn{2}{l|}{ Urubici } & \multicolumn{3}{l|}{ Urupema } \\
\cline { 2 - 9 } & $\mathbf{N}$ & FR & N & FR & N & FR \\
\hline Baixo & 343 & 74,2 & 207 & 87,7 & 187 & 93,0 \\
\hline Médio & 116 & 25,1 & 28 & 11,8 & 14 & 6,9 \\
\hline Alto & 3 & 0,6 & 1 & 0,4 & - & - \\
\hline Prioritário & - & - & - & - & - & - \\
\hline Total & $\mathbf{4 6 2}$ & $\mathbf{1 0 0}$ & $\mathbf{2 3 6}$ & $\mathbf{1 0 0}$ & $\mathbf{2 0 1}$ & $\mathbf{1 0 0}$ \\
\hline
\end{tabular}

Quadro 2 - Grau de atenção para árvores urbanas para as cidades de São Joaquim, Urubici e Urupema, com número e frequência por classe, referente novembro de 2019. Fonte: Autores.

Os indivíduos que apontaram "alto" grau de atenção apresentavam cavidade e fungos no tronco da árvore, indicando o apodrecimento da madeira, galhos podres, levantamento de calçada, copa não característica da espécie (provocada pela realização da poda drástica), infestação por erva-de-passarinho e brotações epicórnicas. Neste caso, recomenda-se a substituição por espécies nativas da região que sejam mais resistentes.

Outro ponto relevante é o grau de infestação de erva-de-passarinho. Em ambas as cidades foram encontradas o hemiparasita, presente na copa das árvores. A infestação em São Joaquim $(38,1 \%)$ e Urubici $(21,2 \%)$, foi superior a constatada em Urupema (1,0\%) (Quadro 3). Em outros trabalhos de Leal et al. (2006) na cidade de Curitiba-PR; de White et al. (2011) em São Cristóvão-SE e Maruyama et al. (2012) em UberlândiaMG, também ocorreu infestação com erva-de-passarinho.
A problemática da presença de erva-de-passarinho está na possível ocupação total das copas, acarretando a redução da eficiência fotossintética levando a um estado de declínio do hospedeiro. Além disso, árvores que apresentam alto grau de infestação estão mais sujeitas ao ataque de insetos e doenças e mais suscetíveis ao estresse ambiental (ARRUDA et al., 2012).

Diante do exposto recomenda-se a poda dos galhos com a infestação de erva-de-passarinho nas três cidades de estudo. Quando a infestação passar dos 50\% da copa sugere-se a substituição do indivíduo. Em contrapartida, Maruyama et al. (2012) aponta que mesmo após a remoção, este hemiparasita pode crescer novamente, no entanto, a poda reduz a produção de sementes, diminuindo sua propagação. Com isso o monitoramento passa a ser um fator relevante, auxiliando na identificação de novos focos de infestação, além de ajudar a traçar planos de manejo para o controle.

Das árvores inventariadas em São Joaquim 50,8\%, apresentaram uma pequena área livre (inferior a $1 \mathrm{~m}^{2}$ ) ou colo soterrado por paver, ou cimento. Em Urubici e Urupema os valores encontrados foram $28 \%$ e $1,5 \%$, respectivamente. $\mathrm{O}$ canteiro pequeno ou a inexistência do mesmo pode dificultar a sobrevivência dos indivíduos arbóreos, pois uma árvore necessita de um espaço de crescimento tanto para a parte aérea, quanto para as raízes (BOBROWSK et al., 2009). Assim, é importante realizar o aumento dessas áreas livres com intuito de promover o melhor desenvolvimento dos indivíduos arbóreos.

\begin{tabular}{|c|c|c|c|c|c|c|c|}
\hline \multirow{2}{*}{ AVALIAÇÃO/VARIÁVEL } & & \multicolumn{2}{|c|}{ São Joaquim } & \multicolumn{2}{|c|}{ Urubici } & \multicolumn{2}{|c|}{ Urupema } \\
\hline & & $\mathbf{n}$ & FR & $\mathbf{n}$ & FR & $\mathbf{n}$ & FR \\
\hline \multirow[t]{3}{*}{ Figueira mata-pau } & $0=$ Ausente & 462 & 100 & 234 & 99,2 & 200 & 99,5 \\
\hline & $1=$ Presente sem raiz no solo & - & - & 2 & 0,8 & 1 & 0,5 \\
\hline & $3=$ Presente com raiz no solo & - & - & - & - & - & - \\
\hline \multirow{3}{*}{$\begin{array}{l}\text { Galhos interferindo } \\
\text { na rede elétrica }\end{array}$} & $0=$ Sem conflito & 450 & 97,4 & 235 & 99,6 & 198 & 98,5 \\
\hline & $1=$ Rede elétrica simples & 12 & 2,6 & 1 & 0,4 & 3 & 1,5 \\
\hline & $3=$ Rede de alta tensão & - & - & - & - & - & - \\
\hline \multirow[t]{3}{*}{ Galhos secos/podres } & $0=$ Sem galhos secos & 114 & 24,6 & 153 & 64,8 & 23 & 11,4 \\
\hline & $2=$ Galhos secos/finos com diâmetro $<12 \mathrm{~cm}$ & 342 & 72,0 & 75 & 31,8 & 177 & 88,1 \\
\hline & $4=$ Galhos secos/finos com diâmetro $>12 \mathrm{~cm}$ & 6 & 1.4 & 8 & 3,4 & 1 & 0,5 \\
\hline \multirow[t]{4}{*}{ Erva-de-passarinho } & $0=$ Ausente & 286 & 61,9 & 186 & 78,8 & 199 & 99,0 \\
\hline & $1=<25 \%$ da copa & 163 & 35,4 & 23 & 9,7 & 2 & 1,0 \\
\hline & $3=25$ a $50 \%$ da copa & 10 & 2,1 & 13 & 5,5 & - & - \\
\hline & $5=>50 \%$ da copa & 3 & 0,6 & 14 & 5,9 & - & - \\
\hline \multirow{2}{*}{$\begin{array}{l}\text { Copa equilibrada/ } \\
\text { Arquitetura natural }\end{array}$} & $0=$ Copa preservada da espécie & 405 & 87,6 & 185 & 78,4 & 116 & 57,7 \\
\hline & $3=$ Copa não característica da espécie & 57 & 12,4 & 51 & 21,6 & 85 & 42,3 \\
\hline
\end{tabular}




\begin{tabular}{|c|c|c|c|c|c|c|c|}
\hline Fungo/Cancro & $0=$ Ausente & 436 & 94.4 & 211 & 89,4 & 164 & 81,6 \\
\hline & $5=$ Presente & 26 & 5,6 & 25 & 10,6 & 37 & 18,4 \\
\hline \multirow[t]{3}{*}{ Cavidade } & $0=$ Ausente & 394 & 85,3 & 213 & 90,3 & 119 & 59,2 \\
\hline & $2=<1 / 3$ do lenho sadio da abertura da cavidade & 56 & 12,1 & 14 & 5,9 & 69 & 34,3 \\
\hline & $4=>1 / 3$ do lenho sadio da abertura da cavidade & 12 & 2,6 & 9 & 3,8 & 13 & 6,5 \\
\hline \multirow[t]{2}{*}{ Cupim } & $0=$ Ausente & 457 & 99,6 & 235 & 99,6 & 200 & 99,5 \\
\hline & $5=$ Presente e/ou vestígios/orifícios do inseto & 2 & 0,4 & 1 & 0,4 & 1 & 0,5 \\
\hline \multirow[t]{2}{*}{ Coleobrocas } & $0=$ Ausente & 444 & 96,1 & 222 & 94,1 & 200 & 99,5 \\
\hline & $5=$ Presente e/ou orifícios & 18 & 3,9 & 14 & 5,9 & 1 & 0,5 \\
\hline \multirow[t]{3}{*}{ Inclinação } & $1=<10 \%$ & 436 & 94,3 & 218 & 92,4 & 183 & 91,0 \\
\hline & $2=10 a 40 \%$ & 24 & 5,3 & 18 & 7,6 & 16 & 8,0 \\
\hline & $4=>40 \%$ & 2 & 0,4 & - & - & 2 & 1,0 \\
\hline \multirow[t]{2}{*}{ Brotações Epicórnicas } & $0=$ Ausente & 288 & 62,3 & 198 & 83,9 & 161 & 80,1 \\
\hline & $2=$ Presente & 174 & 37,7 & 38 & 16,1 & 40 & 19,9 \\
\hline \multirow[t]{2}{*}{ Fungo/Cancro: } & $0=$ Ausente & 376 & 81,4 & 220 & 93,2 & 184 & 91,5 \\
\hline & $5=$ Presente & 86 & 18,4 & 16 & 6,8 & 17 & 8,5 \\
\hline \multirow[t]{4}{*}{ Área livre } & $0=>3 m^{2}$ & 145 & 31,3 & 130 & 55,1 & 141 & 70,1 \\
\hline & $1=1 a 3 m^{2}$ & 78 & 16,9 & 40 & 16,9 & 57 & 28,4 \\
\hline & $2=<1 m^{2}$ & 226 & 48,1 & 24 & 10,2 & 2 & 1,0 \\
\hline & 4 = Sem área livre & 13 & 2,7 & 42 & 17,8 & 1 & 0,5 \\
\hline \multirow{2}{*}{$\begin{array}{l}\text { Levantamento da calçada/ } \\
\text { Afloramento de raiz }\end{array}$} & $0=$ Ausente & 330 & 71,4 & 210 & 89,0 & 199 & 99,0 \\
\hline & $3=$ Presente & 132 & 28,6 & 26 & 11,0 & 2 & 1,0 \\
\hline \multirow[t]{3}{*}{ Circulação dos pedestres } & $1=$ Baixo & 96 & 20,8 & 131 & 55,5 & 119 & 59,2 \\
\hline & $3=$ Médio & 132 & 28,6 & 80 & 33,9 & 7 & 3,5 \\
\hline & $5=$ Alto & 234 & 50,6 & 25 & 10,6 & 75 & 37,3 \\
\hline \multirow[t]{3}{*}{ Circulação de veículos } & $1=$ Baixo & 68 & 14,7 & 132 & 55,9 & 34 & 16,9 \\
\hline & $3=$ Médio & 75 & 16,3 & 71 & 30,1 & 13 & 6,5 \\
\hline & $5=$ Alto & 319 & 69,0 & 33 & 14,0 & 154 & 76,6 \\
\hline
\end{tabular}

Quadro 3 - Avaliação fitossanitária pelo índice de GAAU (Grau de atenção para árvores urbana), com respectivos atributos e valores para as cidades de São Joaquim, Urubici e Urupema (SC) no período de julho a novembro de 2019.

Fonte: Autores.

O índice de Odum foi de 5,77, 4,78 e 4,15 para São Joaquim, Urubici e Urupema, respectivamente. Este índice varia de 2,45 a 26,99 , e o valor encontrado deve estar dentro da escala para ser considerado satisfatório. Porém, os valores encontrados no presente estudo estão próximos do valor mínimo, apontando pouca diversidade (FILHO e BORTOLETO, 2005; ANGELO, 2017).

O índice de diversidade de Odum (d1) é considerado como uma ferramenta de estudo e planejamento da situação atual da arborização urbana, auxiliando na tomada de decisões para órgãos públicos e privados, responsáveis pela manutenção e manejo dessas áreas (BIZ et al., 2015). Ressalta-se que este índice considera o total de espécies em relação ao total de indivíduos, e não a proporção de indivíduos por espécies (ANGELO, 2017).
Valor superior ao encontrado em São Joaquim, Urubici e Urupema foi constatado em trabalho de Bortoleto (2007), avaliando a composição e distribuição da arborização viária da estância de águas de São Pedro (SP) $(19,5)$. Valor inferior ao encontrado no presente estudo foi observado por Biz et al. (2015) na cidade de Dois Vizinhos (PR) $(2,01)$. Ruas com tais índices apresentam maiores complicações em relação ao manejo, pois cada espécie apresenta diferentes comportamentos e necessidades à sobrevivência.

Cabe destacar que quando aplicado os índices de diversidade para as principais ruas e avenidas nas cidades de São Joaquim, Urubici e Urupema, observou-se que das oito ruas avaliadas, sete estão com valores abaixo do que é considerado uma diversidade satisfatória, ou seja 2,45 (BIZ et al., 2015; ANGELO, 2017) (Quadro 4). 
Logo, com a adição do número recomendado de espécies ( $\mathrm{S}$ ad.), o índice de todas as ruas teria uma meIhoria, proporcionando desta forma maior qualidade ambiental para a cidade, bem como, a melhoria da qualidade de vida de seus habitantes e do fornecimento de serviços ecossistêmicos.
Os resultados da espacialização da arborização viária e das árvores nas praças públicas das três cidades estão representados nos mapas temáticos da Figura 3. Em Urupema (Figura $3 \mathrm{~A}$ ), os pontos com maior presença de indivíduos arbóreos/arbustivos foram encontrados na principal rua da cidade, ou seja, na Av. Manoel P. de Medeiros e na praça Manoel Pinto de Arruda, ambas localizadas no bairro Centro.

\begin{tabular}{|l|l|l|l|l|l|l|l|}
\hline Cidade & Nome da rua & $\mathbf{N}^{\circ}$ de espécie atual & $\mathbf{N}^{\circ}$ de exemplares & d atual & S ad. & $\mathbf{N}^{\circ}$ pot & d fut. \\
\hline Urupema & Av. Manoel Pereira & 9 & 83 & $1,81^{*}$ & 8,6 & 50 & 4,2 \\
\hline & Av. Juvelino de Souza & 4 & 10 & $1,30^{*}$ & 11,1 & 123 & 3,8 \\
\hline Urubici & Av. Adolfo Konder & 10 & 24 & $2,83^{*}$ & 3,6 & 109 & 3,4 \\
\hline & Av. Rodolfo Anderman & 4 & 11 & $1,25^{*}$ & 11,4 & 122 & 4,2 \\
\hline São Joaquim & Av. Ivo Silveira & 7 & 61 & $1,46^{*}$ & 10,3 & 72 & 4,1 \\
\hline & R. Manoel Pinto & 5 & 73 & $0,99^{*}$ & 12,7 & 75 & 4,3 \\
\hline & R. Lauro Muler & 12 & 64 & 2,56 & 4,9 & 60 & 4,0 \\
\hline & Av. Irineu Bornhausem & 11 & $2,40^{*}$ & 5,7 & 69 & 4,0 \\
\hline
\end{tabular}

Legenda: *Baixa diversidade.

Quadro 4 - Distribuição do número de espécies e exemplares por rua, índice de diversidade atual (d atual) e futuro (d fut.), número de espécies serem adicionadas ( $\$$ ad.) e número potencial de indivíduos para as principais ruas das três áreas de estudo (SC), no período de julho a novembro de 2019.

Fonte: Autores
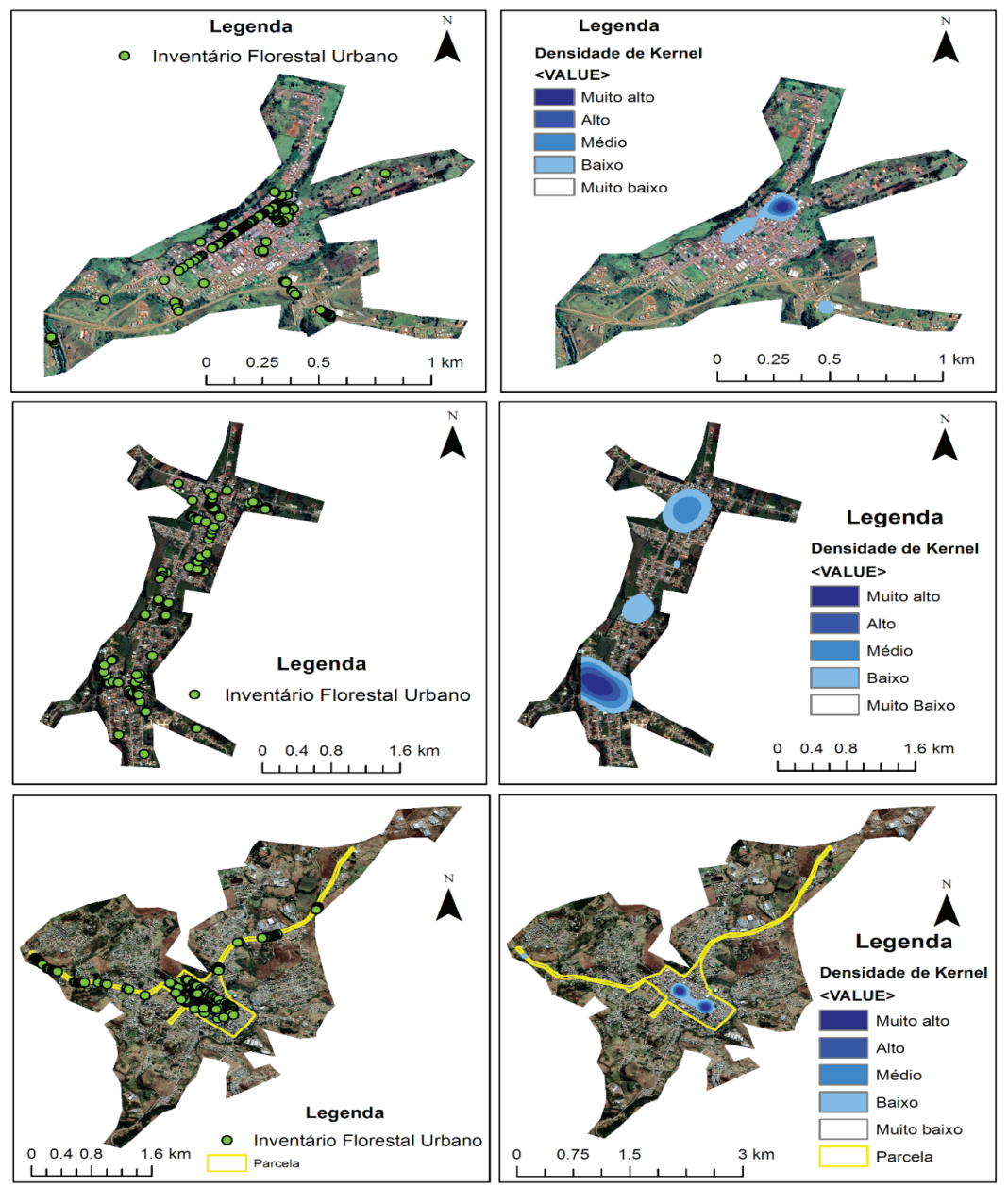

Figura 3 - Mapa temático com a distribuição espacial e estimativa de densidade Kernel dos indivíduos amostrados no inventário florestal urbano. A, $A^{\prime}$ - Urupema; B, $B^{\prime}$ - Urubici e C, $C^{\prime}$ - São Joaquim. No período de julho a novembro de 2019.

Fonte: Autores 
O mesmo comportamento foi encontrado em São Joaquim (Figura 3B'), onde os pontos com maiores densidade de árvores concentraram-se no Centro, nas ruas: Paulo Bathke, Lauro Muller, Ismael Nunes, Marcos Batista; e nas praças João Ribeiro e Cezário Amarante. É importante ressaltar que no ano de 2017, nessas mesmas ruas foi implantado um projeto paisagístico, promovendo o plantio de cinco espécies arbóreas exóticas: $A$. forrestii; $A$. palmatum Atropurpureum; A. palmatum; L. orientalis e L. tulipifera.

A maior concentração de arborização viária no bairro Centro também foi observada em trabalho de Lindenmaier e Souza (2015), na cidade de Cachoeira do Sul (RS) e de Borges et al. (2018) em Patos (PB). Logo, é comum observar que na maioria das cidades brasileiras a distribuição espacial e a frequência de indivíduos arbóreos/arbustivos estejam localizadas em uma determinada região, sendo como uma possível estratégia do poder público em deixar esses locais mais atrativos para os residentes e visitantes. Por outro lado, essa distribuição mais centralizada pode refletir a falta de planejamento urbano voltado para arborização urbana, fazendo-se necessário a elaboração de planos e estratégias que atendam todos os habitantes e não uma pequena parcela.

Em contrapartida, a cidade de Urubici (Figura 3C) apresentou uma distribuição da arborização viária heterogênea entre os bairros, apresentando maior concentração no bairro Centro e Esquina, diferindo da distribuição espacial encontrada em Urupema, a qual está concentrada em um único ponto. A distribuição das árvores em diferentes bairros promove a melhora na qualidade de vida e melhora no embelezamento da cidade para os residentes e visitantes.

De modo geral, a utilização do estimador de Kernel ajudou na interpretação e na percepção da distribuição espacial das árvores inventariadas, demonstrando com maior clareza os locais onde estes ocorrem com maior frequência dando suporte à tomada de decisão para um possível manejo, assim, recomenda-se o plantio de novas árvores nas três cidades envolvendo diferentes bairros, promovendo uma distribuição homogênea dos indivíduos.

Portanto, o uso do banco de dados para arborização de vias públicas é de grande importância, pois fornece informações sobre o entorno de onde o indivíduo arbóreo está inserido, possibilitando realizar cruzamento de informações, além de ser uma aplicação prática, fornecendo diferentes relatórios para o manejo e auxílio ao cadastramento das árvores (FILHO et al., 2002; MAYER et al., 2014).

Para atingir o objetivo de promover a arborização urbana como um novo atrativo turístico para a região da Serra Catarinense, recomenda-se o enriquecimento da arborização com espécies nativas regionais, com apelo paisagístico, aumentando a diversidade da espécie e embelezamento das cidades. Desse modo sugere-se as seguintes espécies:

-Handroanthus albus (Cham.) Mattos (Ipê Amarelo);

-Maytenus muelleri Schwacke (Espinheira-santa);

-Dicksonia sellowiana Hook (Xaxim);

-Acca sellowiana (O.Berg) Burret (Goiaba-serrana);

-Myrrhinium atropurpureum Schott (Carrapato);

- Campomanesia rhombea O.Berg (Guabiroba);

-Vitex megapotamica (Spreng.) Moldenke (Tarumã).

Cabe destacar que a Espinheira-santa quando plantada em pleno sol, a planta perde as características fitoquímicas, havendo um aumento na concentração de taninos na planta (OLIVEIRA et al., 2009). Neste caso a planta em específico é recomenda pela adaptabilidade da região e não pelos fins medicinais como é tradicionalmente conhecida.

O enriquecimento com espécies nativas da região com o verde de alta qualidade e colorido, também foi recomendado para a cidade Savannah, Georgia no Estados Unidos (PIERSKALLA et al., 2016), como estratégia de gestão para manter a cidade atraente ao longo das estações.

Aconselha-se também que os gestores responsáveis pela arborização da cidade, elaborarem e incorporem programas que proporcionem oportunidades para aprender mais sobre as funções, benefícios e exclusividade das florestas urbanas na região da Serra Catarinense, incluindo a educação ambiental e conscientização à natureza. Adicionar florestas urbanas como atrações gratuitas pode não apenas ampliar a versatilidade das ofertas de turismo da cidade, mas também elevar o status da cidade como destino.

\section{CONCLUSÃO}

A metodologia de avaliação do grau de atenção para árvores urbanas, demostrou-se eficaz para o presente estudo, permitindo realizar uma inspeção $360^{\circ}$ na árvore de forma rápida e precisa. Esse modelo foi testado e validado, podendo ser aplicado em outros municípios da Serra Catarinense.

A diversidade foi baixa conforme o índice de diversidade de Odum. A utilização destes índices pode servir de base e motivação para a criação do plano diretor de arborização urbana para as cidades, já que que elas ainda não possuem.

Devem ser realizados novos plantios de árvores com espécies nativas da região, preferencialmente do ecossistema Floresta Ombrófila Mista. Logo, espera-se que esse trabalho possa servir de inspiração e motivação para que novos estudos sejam realizados na região envolvendo a temática da arborização urbana. 


\section{AGRADECIMENTOS}

O presente trabalho foi realizado com apoio da Coordenação de Aperfeiçoamento de Pessoal de Nível Superior-Brasil (CAPES) - Código de Financiamento 001. O trabalho a campo, contou também com parcerias das secretarias municipais de Turismo e Meio Ambiente dos três municípios envolvidos no estudo, sendo São Joaquim, Urubici e Urupema, além do Centro de Treinamento de São Joaquim da Empresa de Pesquisa Agropecuária e Extensão Rural de Santa Catarina - CETREJO/EPAGRI

\section{REFERÊNCIAS}

ALVARES, C. A.; STAPE, J. L.; SENTELHAS, P. C.; GONÇALVES, J. L. M.; SPAROVEK, G. Köppen's climate classification map for Brazil. Meteorologische Zeitschrift. v. 22, p.711-728, 2013.

ANDREATTA, T. R.; BACKES, F. A. A. L.; BELLÉ, R. A.; NEUHAUS, M.; GIRARDI, L. B.; SCHWAB, N. T.; BRANDÃO, B. S. Análise da arborização no contexto urbano de avenidas de Santa Maria, RS. Revista da Sociedade Brasileira de Arborização Urbana, v. 6, n. 1, p. 36-50, 2011.

ANGELO, Dalton Henrique. Índices espaciais e de diversidade florística das zonas central e residencial central de Imperatriz - MA. 2017. 74f. Dissertação (Mestrado em Ciências Florestais e Ambientais) - Universidade Federal do Tocantins, Programa de Pós-Graduação em Ciências Florestais e Ambientais, Gurupi, 2017.

ARAÚJO, J. C.; Fernandes, M. J. C.; da Silva Júnior, O. F. Construções geográficas: teorizações, vivências e práticas. 1.ed. Duque de Caxias - RJ: Espaço científico livre - projetos editoriais, 2013. 184p.

ARRUDA, R.; FADINI, R. F.; CARVALHO, L. N.; DEL-CLARO, K.; MOURÃO, F. A.; JACOBI, C. M.; DETTKE, G. A. Ecology of Neotropical mistletoes: an important canopydwelling component of Brazilian ecosystems. Acta Botanica Brasilica, v. 26, n. 2, p. 264-274, 2012.

Base de dados de espécies exóticas invasoras do Brasil, Instituto Hórus de Desenvolvimento e Conservação Ambiental, Florianópolis - SC. Disponível em: <http:// bd.institutohorus.org.br/www $>$. Acesso em 10 de dez. 2019.

BIZ, S.; PASTÓRIO, A. P.; BRUN, F. G. K.; BRUN, E. J.; WATZLAWICH, L. F. Indicadores de diversidade para a arborização viária do bairro centro norte da cidade de Dois Vizinhos-Paraná. Revista da Sociedade Brasileira de Arborização Urbana, v. 10, n. 3, p. 1-13, 2015.

BOBROWSKI, R.; BIONDI, D.; BAGGENSTOSS, D. Composição de canteiros na arborização de ruas de Curitiba (PR). Revista da Sociedade Brasileira de Arborização Urbana, v. 4, n. 2, p. 44-61, 2009.

BORGES, D. A. B.; LIMA, E. R. V.; DOS SANTOS, J. S.; CUNHA, M. D. C. L.; CUNHA CASTRO, A. A. B. Análise da arborização urbana na cidade de Patos/PB. Revista Brasileira de Geografia Física, v. 11, n. 04, p. 1343-1359, 2018.

BRASIL. Introdução à estatística espacial para a saúde pública. Brasília: Ministério da Saúde, 2007.

BRASIL. Lei ${ }^{\circ} 08$, de 14 de setembro de 2012. Lista Oficial de Espécies Exóticas Invasoras do Estado de Santa Catarina. Diário Oficial da República Federativa do Brasil, Brasília, Brasília, DF, 02 out. 2012. Seção 1, p. 1. DEMUZERE, M.; ORRU, K.; HEIDRICH, O.; OLAZABAL, E.; GENELETTI, D.; ORRU, H.; FAEHNLE, M. Mitigating and adapting to climate change: Multi-functional and multi-scale assessment of green urban infrastructure. Journal of environmental management, v. 146, p. 107-115, 2014. FINI, A.; FERRINI, F.; FRANGI, P.; AMOROSO, G.; PIATTI, R. Withholding irrigation during the establishment phase affected growth and physiology of Norway maple (Acer platanoides) and linden (Tilia spp.). Journal of Arboriculture, v. 35, n. 5, p. 241, 2015.

GAGE, E. A.; COOPER, D. J. Urban forest structure and land cover composition effects on land surface temperature in a semi-arid suburban area. Urban forestry \& urban greening, v. 28, p. 28-35, 2017.

GROHMANN, D.; PETRUCCI, R.; TORRE, L.; MICHELI, M.; MENCONI, M. E. Street trees' management perspectives: Reuse of Tilia sp.'s pruning waste for insulation purposes. Urban forestry \& urban greening, v. 38, p. 177-182, 2018.

IBGE. Manual técnico da vegetação brasileira. Sistema fitogeográfico, inventário das formações florestais e campestres, técnicas e manejo de coleções botânicas, procedimentos para mapeamentos. 2012.

IBGEa, INSTITUTO BRASILEIRO DE GEOGRAFIA E ESTATÍSTICA. São Joaquim - SC. Disponível em: <https://cidades.ibge.gov.br/brasil/sc/sao-joaquim/panorama>. Acesso em: 25 de mar. de 2018.

IBGEb, INSTITUTO BRASILEIRO DE GEOGRAFIA E ESTATÍSTICA. Urubici - SC. Disponível em: <https:// cidades.ibge.gov.br/brasil/sc/urubici/panorama>. Acesso em: 25 de mar. de 2018.

IBGEC, INSTITUTO BRASILEIRO DE GEOGRAFIA E ESTATÍSTICA. Urupema - SC. Disponível em: <https:// cidades.ibge.gov.br/brasil/sc/urupema/panorama>. Acesso em: 25 de mar. de 2018.

IPUF - Instituto de Planejamento Urbano de Florianópolis. Manual Calçada Certa. Florianópolis: 
IPUF, 2 ed. 2019.

LEAL, L.; BUJOKAS, W. M.; BIONDI, D. Análise da infestação de erva-de-passarinho na arborização de ruas de Curitiba, PR. Revista Floresta, Curitiba, v. 36, n. 3, p. 323-330, 2006. LINDENMAIER, D. S.; SOUZA, B. S. P. Avaliação da cobertura vegetal arbórea em Cachoeira do Sul/RS: índice e distribuição espacial do elemento verde na paisagem urbana. Geografia Ensino \& Pesquisa, v. 19, n. 3, p. 79-88, 2015. MARUYAMA, P. K.; MENDES-RODRIGUES, C.; ALVESSILVA, E.; CUNHA, A. F. Parasites in the neighbourhood: Interactions of the mistletoe Phoradendron affine (Viscaceae) with its dispersers and hosts in urban areas of Brazil. Flora-Morphology, Distribution, Functional Ecology of Plants, 207(10), 768-773, 2012.

MAYER, C. L. D.; OLIVEIRA FILHO, P. C.; BOBROWSKI, R. Análise espacial de conflitos da arborização de vias públicas: caso Irati, Paraná. Revista Floresta, v. 45, n. 1, p. 11-20, 2014.

MOURA de SOUSA, L.; FIGUEIRÊDO, M. F.; BRAGA, P. E. T. Levantamento quali-quantitativo da arborização urbana do distrito de Rafael Arruda, Sobral, CE. Revista da Sociedade Brasileira de Arborização Urbana. Piracicaba - SP, v.8, n.3, 2013.

NORTON, B. A.; COUTTS, A. M.; LIVESLEY, S. J.; HARRIS, R. J.; HUNTER, A. M.; WILLIAMS, N. S. Planning for cooler cities: A framework to prioritise green infrastructure to mitigate high temperatures in urban landscapes. Landscape and urban planning, v. 134, p. 127-138, 2015. NOWAK, D. J.; ROWNTREE, R. A.; PHERSON, E. G.; SISINNI, S. M.; KERKMANN, E. R.; STEVENS, J. C. Measuring and analyzing urban tree cover. Landscape and Urban Planning, v. 36, n. 1, p. 49-57, 1996.

ODUM, Eugene. P. FUNDAMENTOS DE ECOLOGIA. Editora Fundação Calouste Gulbenkian, Lisboa, 2001. OLIVEIRA, J. D.; SCIPIONI, M. C.; REIS, A. R. N.; XIMENES, E. Diagnóstico da arborização da Praça Centenário, Curitibanos, Santa Catarina, Brasil. Acta Biológica Catarinense, v. 6, n. 3, p. 23-36, 2019.

SANTOS O. R.; COULAUD C., S.; COLAÇO, W. Revisão da Maytenus ilicifolia Mart. ex Reissek, Celastraceae. Contribuição ao estudo das propriedades farmacológicas. Revista Brasileira de Farmacognosia, v. 19, n. 2B, p. 650-659, 2009.

PIERSKALLA, C. D.; DENG, J.; SINISCALCHI, J. M. Examining the product and process of scenic beauty evaluations using moment-to-moment data and GIS: The case of Savannah, GA. Urban Forestry \& Urban Greening, v. 19, p. 212-222, 2016.

PINHEIRO, R.; FRANCHIN, E.; RIBEIRO, R. S.; WOLFF, W.;
SILVA, A. C.; HIGUCHI, P. Arborização Urbana na Cidade de São José do Cerrito, SC: Diagnóstico e proposta para áreas de maior trânsito. Revista da Sociedade Brasileira de Arborização Urbana, v. 4, n. 4, p. 63-78, 2009.

REDIN, C. G.; VOGEL, C.; TROJAHN, C. D. P.; GRACIOLI, C. R.; LONGHI, S. J. Análise da arborização urbana em cinco praças do município de Cachoeira do Sul, RS. Revista da Sociedade Brasileira de Arborização urbana. Piracicaba - SP, v.5, n.3, 2010. p.149-164.

SAMPAIO, A.C. F. Análise da Arborização de Vias Públicas das Principais Zonas do Plano Piloto de Maringá-PR. 2006. 117 p. Dissertação. (Mestrado em Geografia) Universidade Estadual de Maringá, Maringá, 2006 SANTOS, C. Z. A.; FERREIRA, R. A.; SANTOS, L. R.; SANTOS, L. I.; GOMES, S. H.; GRAÇA, D. A. S. Análise qualitativa da arborização urbana de 25 vias públicas da cidade de Aracajú - SE. Revista Ciência Florestal. Santa Maria - RS, v.25, n.3, 2015.

SANTOS, E M.; SILVEIRA, B. D. B.; SOUZA, A. C.; SCHMITZ, V.; SILVA, A. C.; HIGUCHI, P. Análise quali-quantitativa da arborização urbana em Lages, SC. Revista de Ciências Agroveterinárias, v. 12, n. 1, p. 59-67, 2013.

SANTOS, H. G.; JACOMINE, P. K. T.; ANJOS, L. H. C.; OLIVEIRA, V. A.; LUMBRERAS, J. F.; COELHO, M. R.; ALMEIDA, J. A. ; ARAUJO FILHO, J. C.; OLIVEIRA, J. B.; CUNHA, T. J. F. Sistema Brasileiro de Classificação dos Solos. 5 ed. Brasília: EMBRAPA, 2018.

SILVA, R. V.; ANGELO, D. H.; ARRUDA, A. A.; DA SILVA, W. A. Análise dos principais conflitos e espécies inadequadas presentes na arborização viária na região central do município de Imperatriz (MA). Revista da Sociedade Brasileira de Arborização Urbana, v. 13, n. 2, p. 47-61, 2018.

SJÖMAN, H.; ÖSTBERG, J.; BÜHLER, O. Diversity and distribution of the urban tree population in ten major Nordic cities. Urban Forestry \& Urban Greening, v. 11, n. 1, p. 31-39, 2012.

SOUZA, N. L. Unidades de conservação em áreas urbanas - o caso do parque cinturão verde de Cianorte - modulo Mandhuy. Revista RAEGA. Curitiba - PR, v.23, n.1, 2011. VIBRANS, A. C. Para que inventariar florestas? Reflexões sobre a finalidade do inventário florístico florestal de Santa Catarina. REA - Revista de Estudos Ambientais. Blumenau - SC, v.14, n.1 esp., 2012. p.6-13

WHITE, B. L. A.; RIBEIRO, A. D. S.; WHITE, L. A. S.; NASCIMENTO JÚNIOR, J. E. D. Análise da ocorrência de erva-de-passarinho na arborização da Universidade Federal de Sergipe, Campus de São Cristóvão. Revista Floresta, Curitiba, v. 41, n. 1, 2011. 


\section{AUTORES}

ORCID: https://orcid.org/0000-0002-5594-5584

CHARLES COSTA COELHO, M.Sc. | Universidade Regional de Blumenau (FURB) - Programa de Pós-Graduação em Engenharia Florestal - PPGEF, Blumenau, SC, Brasil. | Correspondência para: R. Barão de Batovi, 587 - Centro, Florianópolis - SC, 88015-340 | email: ccoelho.florestal@ gmail.com

ORCID: https://orcid.org/0000-0002-9537-6275

MARCELO DINIZ VITORINO, Dr. | Universidade Regional de Blumenau (FURB), Programa de Pós - Graduação em Engenharia Florestal - PPGEF, Blumenau, SC, Brasil | Correspondência para: R. São Paulo, 3250 - Itoupava Seca, Blumenau - SC, 89030-000 | email: diniz@furb.br

ORCID: https://orcid.org/0000-0002-3675-925X

BRUNO JUAN GUEDES RODE | Universidade Regional de Blumenau (FURB), Arquitetura, Blumenau, SC, Brasil. |Correspondência para: R. São Paulo, 3250 - Itoupava Seca, Blumenau - SC, 89030-000 | email: brunojuan01@gmail.com

ORCID: https://orcid.org/0000-0001-8557-2443

ANA CAROLINA BOSCHETTI, M.Sc. | Universidade Regional de Blumenau (FURB), Programa de Pós - Graduação em Engenharia Florestal - PPGEF, Blumenau, SC, Brasil. | Correspondência para: R. São Paulo, 3250 - Itoupava Seca, Blumenau - SC, 89030-000 | email: anaboschetti@ hotmail.com

ORCID: https://orcid.org/0000-0001-8557-2443

PAULO AUGUSTO GARBUGIO DA SILVA, M.Sc. | Universidade Federal de Santa Catarina (UFSC), Programa de Pós - Graduação em Biotecnologia e Biociências - PPGBB, Florianópolis, SC, Brasil. | Correspondência para: R. Barão de Batovi, 587 - Centro, Florianópolis - SC, 88015-340 | email: pauloaugustogs@gmail.com

ORCID: https://orcid.org/0000-0001-8557-2443

TATIELE ANETE BERGAMO FENILLI, Dra. | Universidade Regional de Blumenau (FURB), Programa de Pós - Graduação em Engenharia Florestal - PPGEF, Blumenau, SC, Brasil. | Correspondência para: R. São Paulo, 3250 - Itoupava Seca, Blumenau - SC, 89030-000 | e-mail: tfenilli@furb.br

ORCID: https://orcid.org/0000-0003-1078-5641

MARIA RAQUEL KANIESKI, Dra. | Universidade Estadual de Santa Catarina (UDESC), Programa de Pós - Graduação em Engenharia Florestal - PPGEF, Lages, SC, Brasil. |
Correspondência para: Av. Luiz de Camões, 2090 - Conta Dinheiro, Lages - SC, 88520-000 | email: raquel.kanieski@ udesc.br

ORCID: https://orcid.org/0000-0002-8244-5120

KRISTIANA FIORENTIN DOS SANTOS, Dra. | Universidade Regional de Blumenau (FURB), Programa de Pós - Graduação em Engenharia Florestal - PPGEF, Lages, SC, Brasil. | Correspondência para: R. São Paulo, 3250 - Itoupava Seca, Blumenau - SC, 89030-000 | email: kristianafiorentin@ gmail.com

\section{COMO CITAR ESTE ARTIGO}

COELHO, Charles Costa; VITORINO, Marcelo Diniz; RODE, Bruno Juan Guedes; BOSCHETTI, Ana Carolina; SILVA, Paulo Augusto Garbugio da; FENILLI, Tatiele Anete Bergamo; KANIESKI, Maria Raquel; SANTOS, Kristiana Fiorentin dos. Análise Estrutural E Espacial Do Componente Arbóreo Como Elemento De Paisagem Turistíca. MIX Sustentável, [S.I.], v. 7, n. 1, p. 159-170, dez. 2020. ISSN 24473073. Disponível em:<http://www.nexos.ufsc.br/index.php/mixsustentavel>. Acesso em: dia mês. ano. doi:https://doi. org/10.29183/2447-3073.MIX2020.v7.n1.159-170.

DATA DE ENVIO: 02/09/2020

DATA DE ACEITE: 01/12/2020 